\title{
单晶 $\mathrm{Cu}$ 纳米杆拉伸力学特性的尺寸依赖性模拟 *
}

\author{
白清顺 童 振 梁迎春 陈家轩王治国 \\ (哈尔滨工业大学机电工程学院, 哈尔滨 150001)
}

\begin{abstract}
摘 要 对圆形、方形截面形状的单晶 $\mathrm{Cu}$ 纳米杆拉伸变形过程进行了分子动力学模拟. 通过中心对称参数方法并结合位错形 核理论分析了截面形状、截面面积和长细比对纳米杆拉伸力学特性的影响, 研究了单晶 $\mathrm{Cu}$ 纳米杆拉伸力学特性的尺寸依赖性. 结 果表明: 首次屈服后, 纳米杆在 “位错形核 - 位错延伸与滑移 - 晶格原子交叉滑移” 的交替循环作用机制下, 产生塑性变形; 截面 形状对纳米杆拉伸变形的初始塑性影响较小, 而对纳米杆拉伸力学特性的影响较大; 随着截面面积的增大, 两种截面形状的纳米杆 都出现首次屈服点提前, 屈服应力减小和弹性模量增大的现象; 与方形截面形状纳米杆相比, 圆形截面形状纳米杆的屈服应力的变 化率较小, 其弹性模量的变化率较大; 当截面面积增大到 $500 \mathrm{~nm}^{2}$ 后, 两种截面形状纳米杆的弹性模量趋于稳定, 其值接近理论值 $84 \mathrm{GPa}$. 加大仿真规模后, 长细比对纳米杆的拉伸力学特性略有影响.
\end{abstract}

关键词 分子动力学, 拉伸, 纳米杆, 位错形核, 力学特性

中图法分类号 TG113.25, TB303 文献标识码 A 文章编号 0412-1961(2010)10-1173-08

\section{SIMULATION OF SCALE DEPENDENCY ON TENSILE MECHANICAL PROPERTIES OF SINGLE CRYSTAL COPPER NANO-ROD}

\author{
BAI Qingshun, TONG Zhen, LIANG Yingchun, CHEN Jiaxuan, WANG Zhiguo \\ School of Mechatronics Engineering, Harbin Institute of Technology, Harbin 150001 \\ Correspondent: TONG Zhen, Tel: (0451)86413840, E-mail: hit_tz@163.com \\ Supported by National Funds for Distinguished Young Scholars (No.50925521) and National Natu- \\ ral Science Foundation of China (No.50705023) and Natural Scientific Research In- \\ novation Foundation in Harbin Institute of Technology (No.HIT.NSRIF.2009012) and \\ Natural Science Foundation of Heilongjiang Province in China (No.E200903) \\ Manuscript received 2010-06-03, in revised form 2010-08-04
}

\begin{abstract}
The tension process of single crystal $\mathrm{Cu}$ nano-rods with different cross section shapes were simulated by molecular dynamics at atomic scale. Based on centrosymmetry parameter method and combined with the dislocation nucleation theory, the effect of cross-section shape, cross-sectional area and slenderness ratio on the tensile mechanical properties of the nano-rods were analyzed, and the scale dependency of tensile mechanical properties of the single crystal $\mathrm{Cu}$ nano-rods has been studied. The results show that after first yield, the nano-rods produce plastic deformation under the "dislocation nucleation-extended dislocation and sliding-lattice atom cross-slip" mechanism of the alternating cycle. The geometry of cross-section has negligible effects on the tensile initial plasticity of the nano-rods, while it shows apparent effects on the tensile mechanical properties. With the increase of cross-sectional area, two types of nano-rods have the phenomenon of early yield point, yield strength decreases and Young's modulus increases. Compared with that of the square cross-sectional nano-rod, the variable rate of yield stress of the circular cross-sectional nano-rod is smaller and the variable rate of Young's modulus is larger. As the cross-sectional area increases to $500 \mathrm{~nm}^{2}$, the Young's modulus of the two types of nano-rods become stable, and is close to the theoretical value of 84 GPa. Moreover, the slenderness ratio of the nano-rods has a slight effect on the tensile mechanical properties when the
\end{abstract}

* 国家杰出青年科学基金项目 50925521, 国家自然科学基金项目 50705023, 哈尔滨工业大学科研创新基金项目 HIT.NSRIF.2009012 和黑龙江省自然科学基金项目 E200903 资助

收到初稿日期：2010-06-03, 收到修改稿日期： 2010-08-04

作者简介：白清顺, 男, 1974 年生, 副教授, 博士

DOI: 10.3724/SP.J.1037.2010.00266 
simulation size increased.

KEY WORDS molecular dynamics, tension, nano-rod, dislocation nucleation, mechanical property

随着微机械系统 (MEMS) 的发展和纳米机械系统 (NEMS) 的出现 ${ }^{[1]}$, 人们迫切要求深入了解纳米尺度下 材料的力学行为和力学特性. 纳米杆作为纳米器件的基本 构件, 对其力学特性进行详细地研究具有重要意义. 随着 材料尺寸的减小, 材料中缺陷的存在机率降低, 强度提高, 材料的变形机理、力学特性表现出与块体材料不同甚至相 反的特性. Michalske 等 ${ }^{[2]}$ 在表面力显微镜实验中, 发现 基体剪切屈服应力随着探针半径的减小（从 $250 \mathrm{~nm}$ 到 $50 \mathrm{~nm})$ 而增加. Greer 等 ${ }^{[3]}$ 在对直径在微米量级 (0.4$8 \mu \mathrm{m})$ 的单晶 $\mathrm{Au}$ 柱做轴向压缩实验时发现, 单晶 $\mathrm{Au}$ 柱的屈服应力达到了 $\mathrm{GPa}$ 量级, 并且屈服应力随着细柱 体直径的减小而迅速升高. 但由于技术水平及相关实验条 件的限制, 目前实验对象的尺寸与人们的期待仍然有较大 差距.

近年来，原子水平的模拟技术被广泛地应用于纳米 材料力学特性的研究中, 在纳米晶体材料力学行为的分子 动力学模拟方面取得了很多有意义的成果. Komanduri 等 ${ }^{[4]}$ 对纳米尺度下的 $\mathrm{fcc}$ 金属 $(\mathrm{Al}, \mathrm{Cu}$ 和 $\mathrm{Ni})$ 和 $\mathrm{bcc}$ 金 属 $(\mathrm{Fe}, \mathrm{Cr}$ 和 $\mathrm{W})$ 的拉伸变形进行了较全面地研究. 梁海 七等 $[5,6]$ 和徐洲等 ${ }^{[7]}$ 利用分子动力学模拟了 $0 \mathrm{~K}$ 下理 想纳米 $\mathrm{Cu}$ 丝的拉伸变形过程, 揭示了纳米丝的一些变形 过程和机理, 以及纳米丝的应力 - 应变关系、应变率效应、 表面效应和尺寸效应. Doyama 等 ${ }^{[8]}$ 和 Nozaki 等 ${ }^{[9]}$ 采 用嵌入原子势模拟了带有凹坑缺陷的纳米单晶 $\mathrm{Cu}$ 杆的拉 伸、弯曲和压缩过程, 认为缺口底部是应力集中区, 是产生 位错等缺陷的源头. 黄丹等 ${ }^{[10]}$ 研究了单晶 $\mathrm{Ni}$ 纳米丝、纳 米薄膜在 $0 \mathrm{~K}$ 下的准静态拉伸破坏过程, 结果表明 $\mathrm{Ni}$ 纳 米薄膜的断裂接近脆性断裂, 而 $\mathrm{Ni}$ 纳米丝在断裂过程表 现出微弱的塑性. 梁迎春等 ${ }^{[11]}$ 利用分子动力学模拟了纳 米加工后纳构件的拉伸过程, 通过分析、计算获得了非理 想纳米构件的拉伸力学特性. Tschopp 和 McDowell ${ }^{[12]}$ 模拟了单晶 $\mathrm{Cu}$ 纳米杆的单向拉伸与压缩变形, 并研究了 温度和应力对单晶 $\mathrm{Cu}$ 内部位错形核影响. 张宇等 ${ }^{[13]}$ 研 究了 $0 \mathrm{~K}$ 下纳米双晶 $\mathrm{Cu}$ 杆在单向拉伸载荷作用下弹性 性能的应变率效应和尺寸效应.

上述实验和数值模拟结果表明，晶体材料的塑性行 为具有很强的尺寸依赖性. 然而, 由于仿真水平的限制, 前 述研究中多考虑方形截面形状和小尺寸的纳米杆, 而对圆 形截面形状和大尺寸的纳米杆研究较少. 为了深入研究单 晶 $\mathrm{Cu}$ 纳米杆的拉伸力学特性, 本文建立了多种规模下的 圆形、方形截面形状的纳米杆模型，通过分析、计算获得 了纳米杆的力学特性, 进而分析了截面形状、截面面积和 长细比对纳米杆力学性能的影响, 揭示了纳观尺度下单晶
$\mathrm{Cu}$ 纳米杆拉伸变形的尺寸依赖性.

\section{1 模拟方法}

\section{1 模拟模型的建立}

圆形、方形截面纳米杆的初始构型按几何方法生成, 如图 1 所示. $x, y$ 和 $z$ 坐标轴分别对应 fcc 的 [100], [010] 和 [001] 晶向; 横截面为 $y z$ 面, $x$ 轴正向为拉伸方向, 原 子位置按理想点阵排列. 为获得与实际纳米杆相同的仿真 结果, 模型建立时各表面均采用自由边界条件, 同时, 在拉 伸前对每组模型均进行充分的弛豫过程, 弛豫后的纳米杆 形貌如图 $1 \mathrm{~d}$ 和图 1e 所示. 为使纳米杆在拉伸过程中处 于平衡状态, 拉伸应变速率均取 $4.610 \times 10^{8} \mathrm{~s}^{-1}$.

(a)
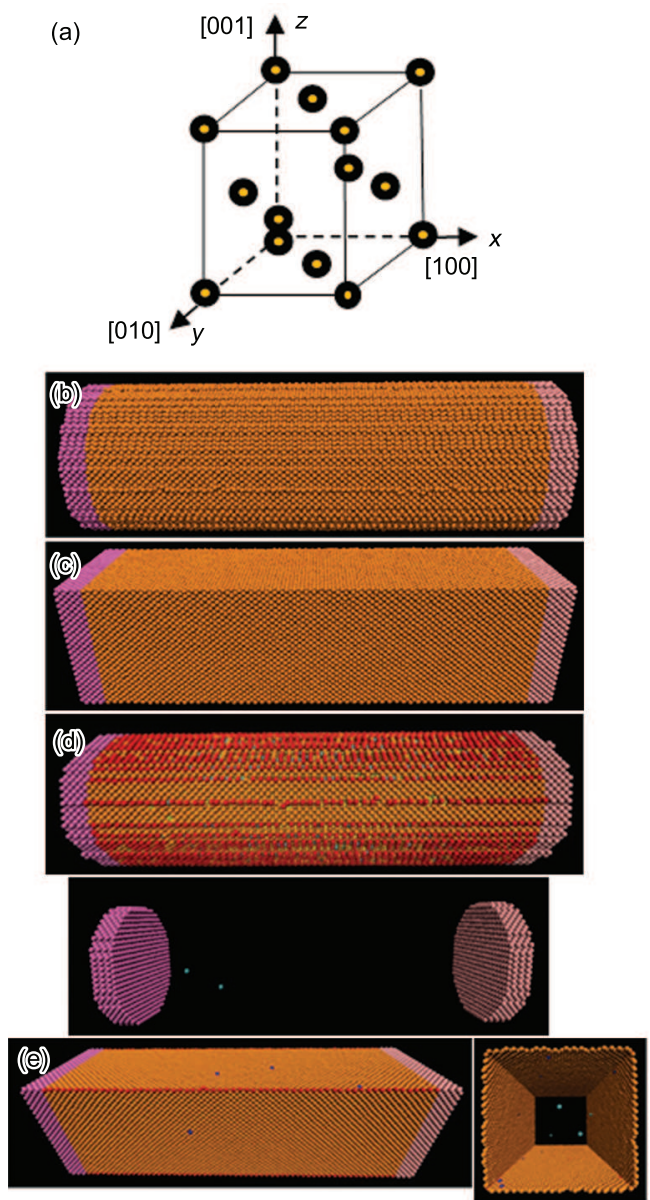

图 1 单晶 $\mathrm{Cu}$ 纳米杆模型建立

Fig.1 Initial configuration of copper nano-rod
(a) schematic of reference frame
(b) copper nano-rod with circular cross section
(c) copper nano-rod with square cross section
(d) copper nano-rod with circular cross section after relaxtion
(e) copper nano-rod with square cross section after relaxtion 


\section{2 仿真参数设置}

$\mathrm{Cu}$ 原子之间的相互作用采用以下两式所示的 EAM 嵌入原子势 ${ }^{[14]}$ 描述:

$$
\begin{gathered}
U=\sum_{i} F_{i}\left(\rho_{i}\right)+\frac{1}{2} \sum_{j \neq i} \phi_{i j}\left(r_{i j}\right) \\
\rho_{i}=\sum_{j \neq i} \rho_{j}\left(r_{i j}\right)
\end{gathered}
$$

式中, $U$ 为系统的总能量, $\rho_{i}$ 是除第 $i$ 个原子以外的所有 其它原子的核外电子在第 $i$ 个原子处产生的电子云密度 之和, $r_{i j}$ 是第 $i$ 个原子与第 $j$ 个原子之间的距离. $F_{i}\left(\rho_{i}\right)$ 是嵌入能; $\phi_{i j}\left(r_{i j}\right)$ 是对势项, $\rho_{j}\left(r_{i j}\right)$ 为电子密度的分布 函数.

$$
\begin{aligned}
& \text { 系统原子的 Virial 应力 } \prod^{\alpha \beta[15]} \text { 定义为 } \\
& \prod^{\alpha \beta}=\frac{1}{\Omega}\left(-\sum_{i} m_{i} v_{i}^{\alpha} v_{i}^{\beta}+\frac{1}{2} \sum_{i} \sum_{j \neq i} F_{i j}^{\alpha} r_{i j}^{\beta}\right)
\end{aligned}
$$

式中, $\Omega$ 为系统体积; $m_{i}$ 和 $v_{i}$ 为原子 $i$ 的质量和速度; $F_{i j}^{\alpha}$ 为原子 $i$ 与原子 $j$ 之间的作用力; $\alpha, \beta$ 为 Cartesian 坐标分量指标; $r_{i j}^{\beta}$ 为原子间距向量 $\vec{r}_{i j}\left(=r_{i}-r_{j}\right)$ 沿着 $\beta$ 坐标的投影.

通过分解 Virial 应力获得原子 $i$ 的原子级应力 $\prod_{i}^{\alpha \beta}$

$$
\begin{gathered}
\prod_{i}^{\alpha \beta}=\frac{1}{\Omega} \sum_{i} \omega_{i} \sigma_{i}^{\alpha \beta} \\
\sigma_{i}^{\alpha \beta}=\frac{1}{\omega_{i}}\left(-m_{i} v_{i}^{\alpha} v_{i}^{\beta}+\frac{1}{2} \sum_{j \neq i} F_{i j}^{\alpha} r_{i j}^{\beta}\right)
\end{gathered}
$$

式中, $\sigma_{i}^{\alpha \beta}$ 为原子 $i$ 的原子级应力, $\sum \omega_{i}=\Omega, \omega_{i}$ 为原子 $i$ 的有效体积.

数值积分采用速度 Verlet 算法 ${ }^{[16]}$, 通过 NoseHoover 方法 ${ }^{[17,18]}$ 将温度调节至 $293 \mathrm{~K}$, 加载过程中 采用速度标定法使系统保持恒定的温度 $293 \mathrm{~K}$. 拉伸过 程中原子内部缺陷采用中心对称参数 (Centrosymmetry Parameter $)^{[19]}$ 来识别. 计算原子 $i$ 的中心对称参数为

$$
\rho_{i}=\sum_{i=1}^{6}\left|\vec{R}_{i}+\vec{R}_{i+6}\right|^{2}
$$

式中, $\vec{R}_{i}$ 和 $\vec{R}_{i+6}$ 为 fcc 晶格相对最近原子的 6 对向量. 本文中原子缺陷结构识别的约定如表 1 所示.

晶体结构的无序化程度采用径向分布函数（radial distribution function, RDF) 来表征.

\section{2 不同截面形状的纳米杆拉伸过程仿真}

为了研究截面形状对纳米杆拉伸力学特性的影响, 设 定两种截面形状纳米杆的长度与截面面积相同, 其中, 纳
米杆长 $L=60 a_{0}$, 圆形截面半径 $R=11 a_{0}$, 方形截面边长 $A=20 a_{0}\left(a_{0}=0.3615 \mathrm{~nm}\right.$, 为 $\mathrm{Cu}$ 单晶的晶格常数).

纳米杆整个拉伸过程的应力 - 应变曲线如图 2 所示, 根据纳米杆变形的不同形态, 将整个拉伸过程分为 3 个阶 段: 弹性变形阶段 ( $\mathrm{OA}$ 段)、塑性延展阶段 ( $\mathrm{AC}$ 段) 和断 裂阶段 (CD 段). 具有不同截面形状的纳米杆应力 - 应变 曲线的整体变化趋势基本一致. 在弹性变形阶段, 纳米杆 的应力 - 应变呈线性关系, 可以求得圆形、方形截面形状 纳米杆的弹性模量分别为 71.6 和 $80.1 \mathrm{GPa}$. 这表明在该 仿真尺度下, 方形截面纳米杆抗变形能力比圆形截面纳米 杆强, 这与宏观尺度下纳米杆的力学特性有所不同. 在 A 点 $(\varepsilon=0.090)$, 两种截面形状的纳米杆的应力同时达到最 大值, 可将其定义为纳米杆的初始屈服应力. 由图可以看 出, 方形截面形状的纳米杆的初始屈服应力明显大于圆形 截面形状的纳米杆. 随着应变的持续加载, 两种纳米杆的 应力 - 应变曲线呈锯齿状波动下降.

图 3 和 4 分别为圆形、方形截面形状的纳米杆在拉 伸过程中的缺陷原子演化图. 由图 3a 图 4a 可以看出, 具 有不同截面形状的纳米杆在弛豫完成后, 自由表面的原子 由于表面张力的作用, 严重偏离理想晶格位置, 如图中所 示纳米杆表面的红色和橙色原子. 在弹性变形阶段, 纳米 杆原子的晶格按比例线性伸长, 变形为弹性变形; 纳米杆 内部无位错产生, 仅有少量的原子为了达到局部的能量平 衡而移动, 从而产生少量空位. 在弹性变形后期, 表层原

\section{表 1 中心对称参数缺陷识别约定}

Table 1 The default value of atomic defects structure in centrosymmetry parameter

\begin{tabular}{ccc}
\hline Lattice structure & $P_{i}$ & Represent color \\
\hline Ideal fcc structure & $P<3$ & Yellow \\
Partial dislocation & $3<P<5$ & Cyan \\
Stacking fault & $5<P<9$ & Blue \\
Surface atom & $9<P<20$ & Orange \\
Whole dislocation & $P>20$ & Red \\
\hline
\end{tabular}

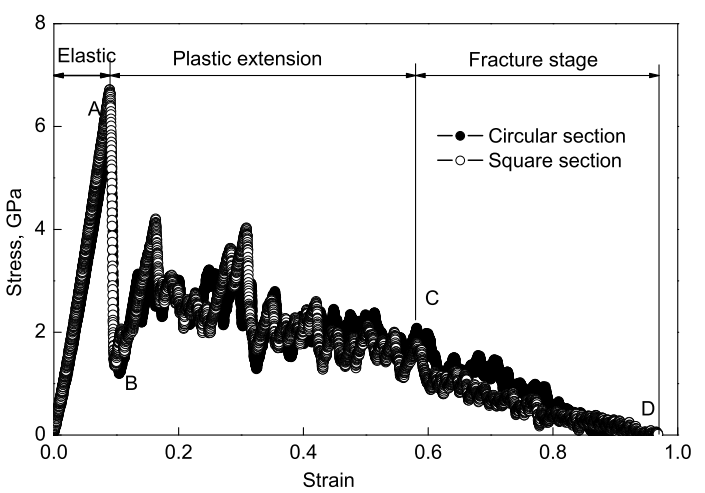

图 2 圆形、方形截面形状单晶 $\mathrm{Cu}$ 纳米杆拉伸应力 - 应变曲线

Fig.2 Tension stress-strain curves of single crystal copper nano-rod with circular and square cross section shapes 


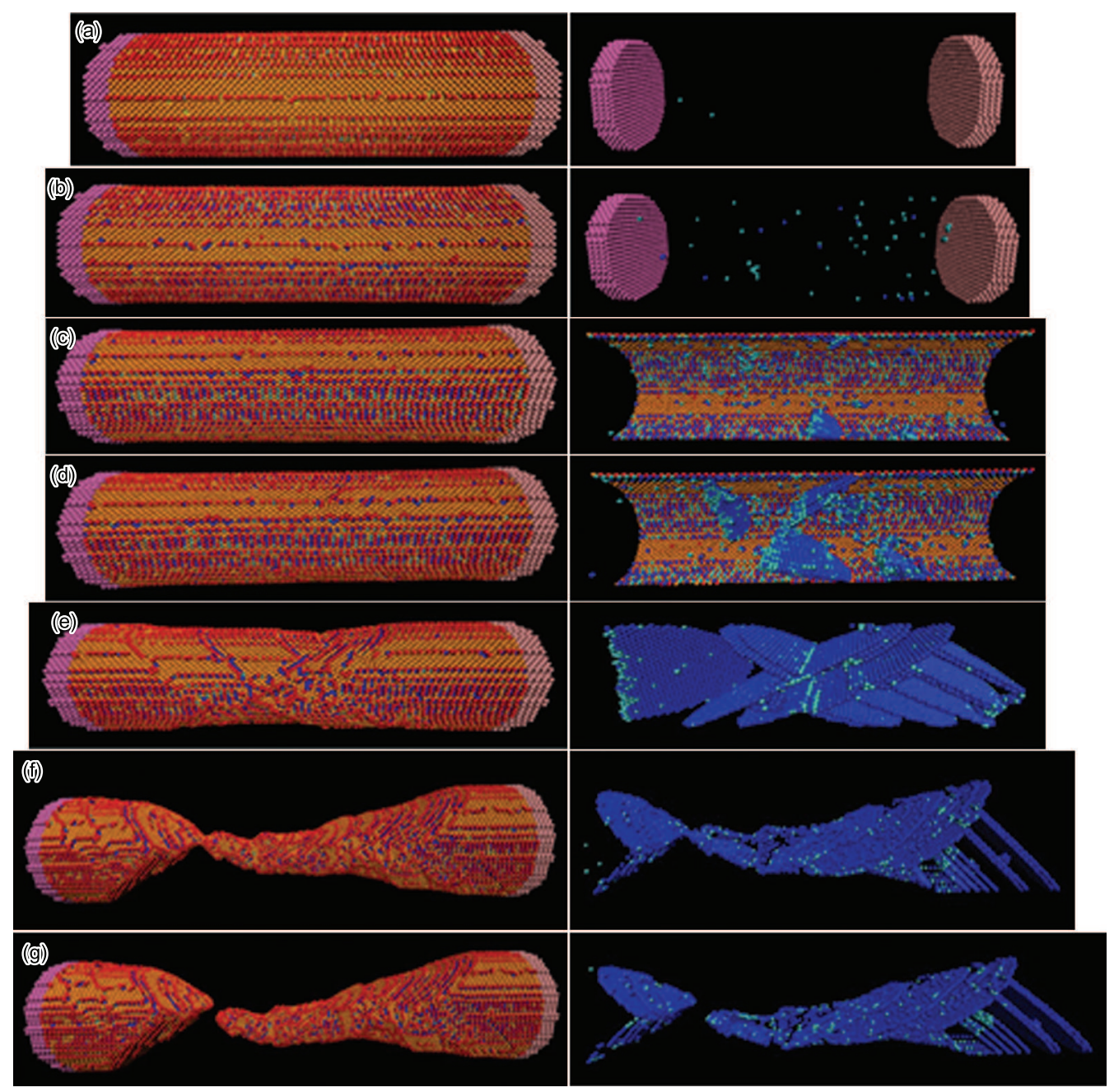

图 3 圆形截面形状纳米杆拉伸变形的原子结构演化图

Fig.3 Evolution of defects in circular cross-section nano-rod during tensile process
(a) $\varepsilon=0$
(b) $\varepsilon=0.089$
(c) $\varepsilon=0.09$
(d) $\varepsilon=0.092$
(e) $\varepsilon=0.106$
(f) $\varepsilon=0.857$
(g) $\varepsilon=0.870$

子缺陷运动加剧, 同时, 纳米杆内部间隙原子数量逐渐增 多, 少量空穴聚集一起形成空位簇且原子迁移情况明显增 加, 如图 $3 \mathrm{~b}$ 和 $4 \mathrm{~b}$ 中所示的蓝色和绿色原子.

由图 3c 和 $4 \mathrm{c}$ 可以看出, 在 $\varepsilon=0.09$ 时, 两种纳米杆 模型的自由表面均有位错开始形核, 纳米杆进入首次屈服 阶段. 随着应变的持续增加，位错在自由表面多处形核并 沿着 $\{111\}$ 滑移系滑移. 纳米杆拉伸积累的能量在位错运 动时被释放, 纳米杆应力迅速下降. 这主要是由于在纳米 尺度下, 位错滑移的空间有限, 很快整个滑移面开始移动. 在应力 - 应变曲线上的表现为: 当达到峰值应力以后, 应 力会发生突降, 然后在一个较小的应力水平附近震荡 (如 图 2 所示 $\mathrm{AB}$ 段). 上述现象表明, 位错形核是发生在塑 性流动之前, 在纳米尺度下, 金属单晶材料的初始屈服是 由位错形核决定的. 这种现象也证实了国内外相关学者对 单晶 $\mathrm{Cu}$ 力学特性的研究结果 $[11,20]$.

首次屈服以后, 位错环逐渐扩展形成多个完整的堆垛 层错面, 并在纳米杆表面生成位错运动的台阶, 如图 $3 \mathrm{e}$ 和
$4 \mathrm{e}$ 所示. 当应力累积到一定值后, 大量新的位错在滑移面 上繁殖, 并在滑移面的交界处造成位错塞积, 堆垛层错相 互交错阻止了位错的进一步扩展, 使得应力有所回升. 当 应力上升到足以驱动位错运动时, 位错开始延伸, 部分晶 格原子沿滑移面进行交叉滑移. 此过程表现为应力 - 应变 曲线呈锯齿状波动下降 (如图 2 所示 $\mathrm{BC}$ 段). 纳米杆在 “位错形核 - 位错延伸与滑移 - 晶格原子交叉滑移” 的交 替循环作用机制下，产生塑性延展. 随着应变的持续加载, 纳米杆长度逐渐伸长, 直径逐渐缩小, 纳米杆内部晶格缺 陷集中的部位开始产生类似宏观的 “颈缩” 现象, 如图 $3 \mathrm{f}$ 和 $4 \mathrm{f}$ 所示.

图 5 为断裂前期 $(\varepsilon=0.825)$, 两种截面形状纳米杆的 径向分布函数图. 由图可以看出, 在断裂前期, 纳米杆的 有序性明显降低, 尤其是短程有序性明显降低, 同时长程 有序性也表现为逐步无序状态. 结合拉伸过程的瞬间原子 演化图 (图 $3 \mathrm{f}$ 和 $4 \mathrm{f}$ ) 和径向分布函数可以看出, 纳米杆在 断裂阶段, “颈缩” 区的部分晶格原子呈现非晶态, 随着应 


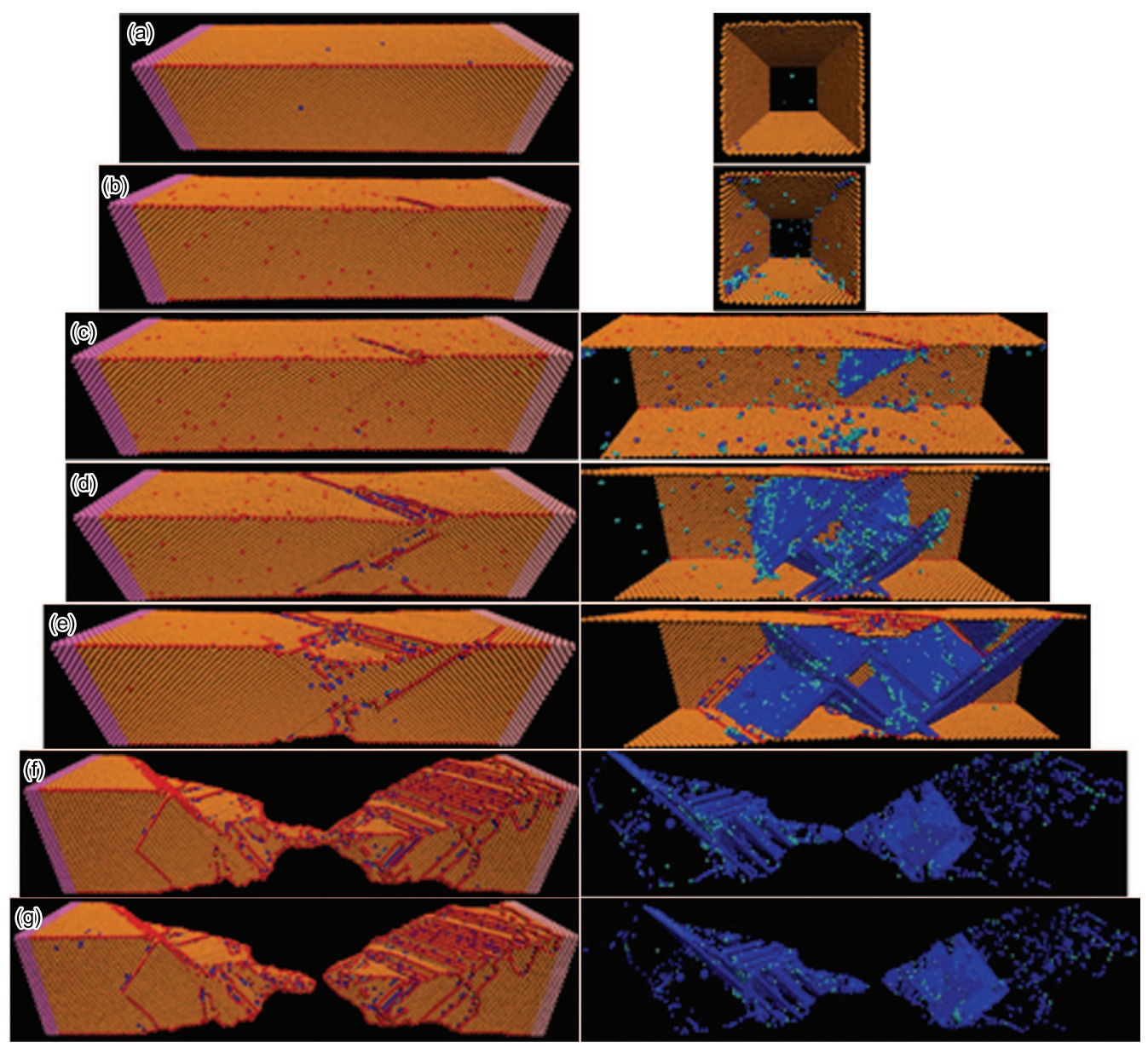

图 4 方形截面形状纳米杆拉伸变形的原子结构演化图

Fig.4 Evolution of defects in square section copper nano-rod during tensile process
(a) $\varepsilon=0$
(b) $\varepsilon=0.089$
(c) $\varepsilon=0.09$
(d) $\varepsilon=0.092$
(e) $\varepsilon=0.101$
(f) $\varepsilon=0.917$
(g) $\varepsilon=0.941$

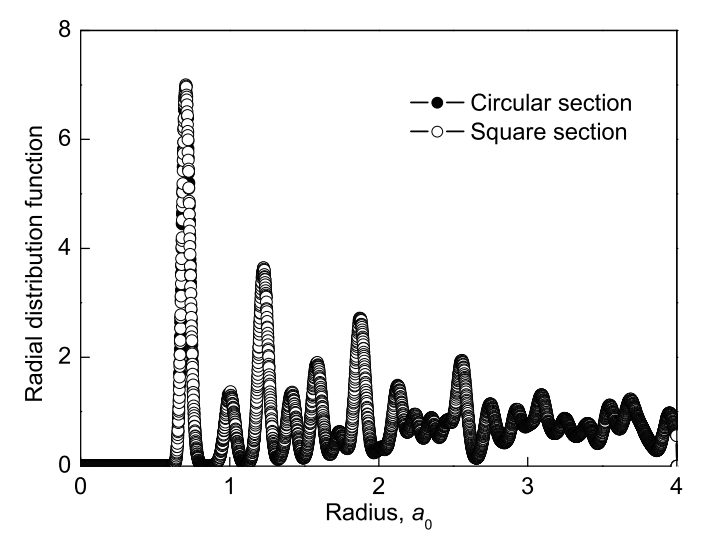

图 5 纳米杆断裂前期径向分布函数图 $(\varepsilon=0.825)$

Fig.5 Radial distribution function of nano-rod with cross section at $\varepsilon=0.825$

变的持续加载, “颈缩” 区中间部分的原子间距变大, 大量 原子键断裂, 纳米杆直径急剧变细, 最终实现纳米杆的断 裂 (图 $3 \mathrm{~g}$ 和 $4 \mathrm{~g}$ ).

\section{3 截面面积对纳米杆拉伸力学特性的影响}

为深入研究截面面积对纳米杆拉伸力学特性的影响
规律, 计算得出了如表 2 和 3 所示的 12 组数据. 计算中, 圆形和方形截面形状的纳米杆长度均为 $L=60 a_{0}$. 表 2 和 3 中, $A$ 代表方形截面边长, $R$ 代表圆形截面半径. 初始 弹性模量为 $0.000<\varepsilon<0.021$ 范围的最小二乘法的线性 拟合值, 后期弹性模量为 $0.030<\varepsilon<\varepsilon_{\sigma s}$ 范围的最小二 乘法的线性拟合值 (其中, $\varepsilon_{\sigma s}$ 为屈服点对应的应变).

\section{1 截面面积对屈服强度的影响}

从表 2 和 3 可知, 随着截面面积的增大, 两种截面形 状的纳米杆均出现屈服应变提前, 屈服强度降低的现象. 这种相类似的仿真结果在其它分子水平的模拟 ${ }^{[7,10]}$ 和微 纳尺度的实验中 ${ }^{[2,3]}$ 也有所发现, 这也说明尺寸效应是微 纳米尺度下 fcc 金属晶体材料的普遍属性.

Hirth 等 ${ }^{[21]}$ 曾经研究过位错形核理论并给出了位 错环形成的自由能 $\Delta G$ 的表达式

$$
\begin{aligned}
& \Delta G=2 \pi r W-\pi r^{2} b \tau+\pi r^{2} \gamma \\
& W=\frac{2-v}{2(1-v)} \frac{G b^{2}}{4 \pi}\left(\ln \frac{4 r}{r_{0}}-2\right)
\end{aligned}
$$

式中, $W$ 是位错环线能, $r$ 是位错环半径, $\tau$ 是作用在位 
表 2 圆形截面形状纳米杆模型尺寸及计算结果

Table 2 Size parameters and simulation results of circular cross section nano-rod

\begin{tabular}{ccccccc}
\hline Group & 1 & 2 & 3 & 4 & 5 & 6 \\
\hline$R / a_{0}$ & 11 & 17 & 23 & 28 & 34 & 40 \\
Real size, $\mathrm{nm}^{2}$ & 50 & 118 & 217 & 322 & 474 & 656 \\
Surface atoms, \% & 5.9 & 4.2 & 3.3 & 2.9 & 2.5 & 2.3 \\
Yield strength, & 5.94 & 6.42 & 6.22 & 6.07 & 5.63 & 5.77 \\
GPa & & & & & & \\
Yield point & 0.090 & 0.090 & 0.087 & 0.084 & 0.077 & 0.076 \\
$\begin{array}{c}\text { Initial Young's } \\
\text { modulus, GPa }\end{array}$ & 54.32 & 60.94 & 64.07 & 66.99 & 71.23 & 76.00 \\
$\begin{array}{c}\text { Finial Young's } \\
\text { modulus, GPa }\end{array}$ & 71.61 & 76.54 & 78.53 & 79.80 & 82.72 & 84.01 \\
\hline
\end{tabular}

表 3 方形截面形状纳米杆模型尺寸及计算结果

Table 3 Size parameters and simulation results of square section nano-rod

\begin{tabular}{ccccccc}
\hline Group & 1 & 2 & 3 & 4 & 5 & 6 \\
\hline$A / a_{0}$ & 20 & 30 & 40 & 50 & 60 & 70 \\
Real size, $\mathrm{nm}^{2}$ & 52 & 118 & 209 & 327 & 470 & 640 \\
$\begin{array}{c}\text { Surface atoms, \% } \\
\text { Yield strength, }\end{array}$ & 2.3 & 1.8 & 1.6 & 1.5 & 1.4 & 1.3 \\
$\quad$ GPa & & 5.90 & 5.12 & 4.72 & 4.06 & 3.92 \\
$\begin{array}{c}\text { Yield point } \\
\text { Initial Young's }\end{array}$ & 0.089 & 0.080 & 0.070 & 0.065 & 0.060 & 0.055 \\
$\begin{array}{c}\text { modulus, GPa } \\
\text { Finial Young's }\end{array}$ & 80.09 & 80.12 & 81.23 & 82.72 & 82.91 & 84.12 \\
modulus, GPa & & 65.46 & 68.40 & 72.20 & 76.18 \\
\hline
\end{tabular}

错环上的剪切应力, $b$ 是 Burgers 矢量幅值, $\gamma$ 是堆垛层 错能, $G$ 是剪切模量, $v$ 是 Poisson 比, $r_{0}$ 是位错核半径.

位错形核的条件是作用在位错环上的剪切应力所作 的功刚好等于形成位错环所需要的能量. 将式 (8) 代入式 (7), 并令 $\Delta G=0$, 即可得到临界剪切应力 $\tau_{\mathrm{c}}$

$$
\tau_{\mathrm{c}}=\frac{1}{r} \frac{2-v}{1-v} \frac{G b}{4 \pi}\left(\ln \frac{4 r}{r_{0}}-2\right)+\frac{\gamma}{b}
$$

式 (9) 表明, 塑性流动之前, 如果形成的位错环较大, 那么只需要施加较小的剪切应力就可以使其开动.

由前面对纳米杆拉伸过程中原子缺陷结构的研究可 知, 在纳米尺度下, 不同截面形状纳米杆的初始塑性均表 现为位错首先在纳米杆表面形核, 金属单晶材料的初始屈 服主要与位错形核有关. 因此, 初始屈服应力的尺寸效应 实际上是源于材料在位错形核过程中受其外观几何尺寸 的影响. 在纳米杆弹性变形的后期, 位错大量形核, 由于 较大的截面面积的纳米杆会为形成较大位错环提供更多 的空间和机会, 所以能在较小屈服应力下形成位错核, 完 成首次屈服. 此外, Michalske 等 ${ }^{[2]}$ 曾通过实验分析认
为, 随着空间的增大, 与形成一个大位错环相比, 形成多个 小位错环要容易得多. 这表明在相同的应力应变条件下, 较大截面面积的纳米杆内部位错形核的数量多. 因此, 增 大截面面积后, 纳米杆屈服应力降低, 屈服应变减小.

图 6 为纳米杆表面原子比例随截面面积变化图, 由 图可以看出, 随着截面面积的增大, 纳米杆表面原子比例 逐渐减小, 尤其当截面面积增大到 $500 \mathrm{~nm}^{2}$ 后, 两种纳米 杆的表面原子比例均大大减小且变化较小.

图 7 为两种截面形状纳米杆的屈服应力随截面面积 变化图. 随着截面面积的增加, 圆形截面形状纳米杆的屈 服应力具有先增后减的过程, 并与方形截面形状纳米杆的 屈服应力有交叉点，随着截面面积的进一步增大，其屈服 强度的变化率较小, 屈服强度逐渐大于方形截面形状纳米 杆. 前面我们已分析得到, 初始屈服应力的尺寸效应实际 上是源于材料在位错形核过程中受其外观几何尺寸的影 响. 虽然两种纳米杆的长度和截面面积均相同, 但是两者 的外观几何特征却不相同 (例如, 在相同长度和截面面积 下, 两种纳米杆的表面原子比例不同, 如图 6 所示), 这使 两种纳米杆的屈服强度产生了差异.

\section{2 截面面积对弹性模量的影响}

由图 8 可以看出, 随着截面面积的增大, 两种截面形 状的纳米杆弹性模量均增大, 这与实验 ${ }^{[22]}$ 观察到的纳米 $\mathrm{Ag}$ 薄膜的弹性模量随厚度变化的趋势基本一致. 同时, 同 一截面面积下, 方形截面形状纳米杆的初始弹性模量和后 期弹性模量均大于圆形截面形状纳米杆, 但其弹性模量随 截面面积的变化率小于圆形截面形状纳米杆. 当截面面积 增大到 $500 \mathrm{~nm}^{2}$ 后 $\left(A \geq 60 a_{0}, R \geq 34 a_{0}\right)$, 两种截面形 状的纳米杆弹性模量基本相等并接近理论值 $84 \mathrm{GPa}$. 结 合图 6 可知, 在小规模尺度下, 表面效应对纳米杆力学特 性影响大, 两种纳米杆表面原子比例的差异能够对弹性模 量产生较大的影响; 当截面尺寸增大到一定值后, 虽然不 同截面形状纳米杆的表面原子比例仍然存在差异, 但由于 此仿真尺度下, 表面原子比例已经很小, 其影响可以忽略

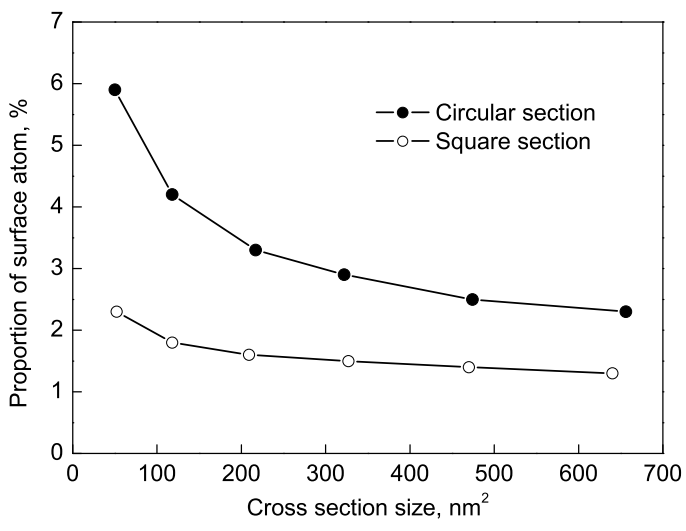

图 6 表面原子比例随截面面积变化曲线

Fig.6 The proportion of surface atoms as a function of cross-sectional area 


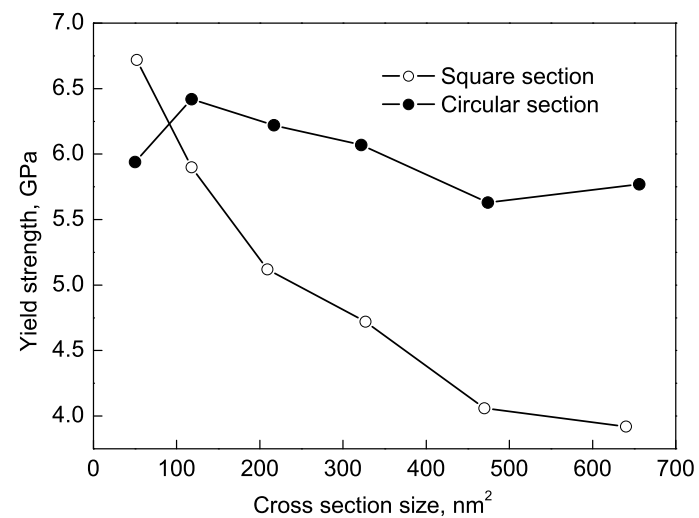

图 7 屈服强度随截面面积变化曲线

Fig.7 Yield strength as a function of cross-sectional area
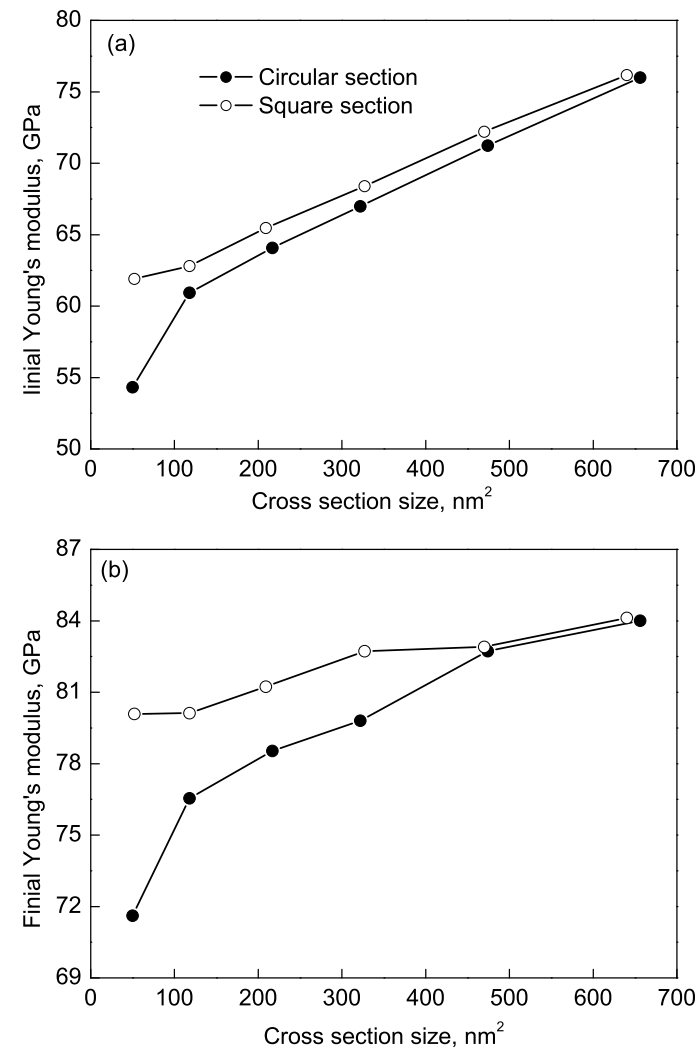

图 8 弹性模量随截面面积变化曲线

Fig.8 Young's modulus as a function of cross-sectional area (a) Initial Young's modulus (b)Finial Young's modulus

不计, 故两种纳米杆的弹性模量也逐渐趋近于稳定值.

\section{4 长细比对纳米杆拉伸力学特性的影响}

为进一步分析不同规模尺寸下长细比对纳米杆弹性 模量和屈服强度的影响, 本文加大了仿真规模, 计算了如 表 4 和 5 所示 10 组数据.

由图 9 可以看出, 加大仿真规模后, 随着长细比的增 大, 具有不同截面形状的纳米杆的屈服应力和弹性模量均 减小, 弹性模量出现明显的“软化”现象. 该仿真结果与
表 4 圆形截面形状纳米杆仿真参数及计算结果

Table 4 Size parameters and simulation results of circular cross section nano-rod

\begin{tabular}{cccccc}
\hline Group & 1 & 2 & 3 & 4 & 5 \\
\hline Length of nano-staff/ $a_{0}$ & 40 & 60 & 80 & 100 & 120 \\
$R / a_{0}$ & 11 & 11 & 11 & 11 & 11 \\
Slenderness ratio & $2: 1$ & $3: 1$ & $4: 1$ & $5: 1$ & $6: 1$ \\
Yield strength, GPa & 6.12 & 5.99 & 5.91 & 5.89 & 5.90 \\
Yield point & 0.091 & 0.091 & 0.090 & 0.091 & 0.090 \\
Initial Young's & 56.07 & 53.51 & 53.07 & 52.74 & 52.49 \\
modulus, GPa & & & & & \\
Finial Young's & 71.77 & 70.99 & 70.40 & 70.17 & 69.89 \\
modulus, GPa & & & & & \\
\hline
\end{tabular}

表 5 方形截面形状纳米杆仿真参数及计算结果

Table 5 Size parameters and simulation results of square section nano-rod

\begin{tabular}{cccccc}
\hline Group & 1 & 2 & 3 & 4 & 5 \\
\hline Length of nano-staff/ $a_{0}$ & 40 & 60 & 80 & 100 & 120 \\
$A / a_{0}$ & 20 & 20 & 20 & 20 & 20 \\
Slenderness ratio & $2: 1$ & $3: 1$ & $4: 1$ & $5: 1$ & $6: 1$ \\
Yield strength, GPa & 6.83 & 6.78 & 6.65 & 6.45 & 6.39 \\
$\quad$ Yield point & 0.090 & 0.091 & 0.089 & 0.087 & 0.088 \\
Initial Young's & 63.90 & 61.56 & 61.52 & 60.11 & 58.79 \\
modulus, GPa & & & & & \\
Finial Young's & 81.84 & 80.62 & 80.04 & 79.68 & 77.73 \\
modulus, GPa & & & & & \\
\hline
\end{tabular}
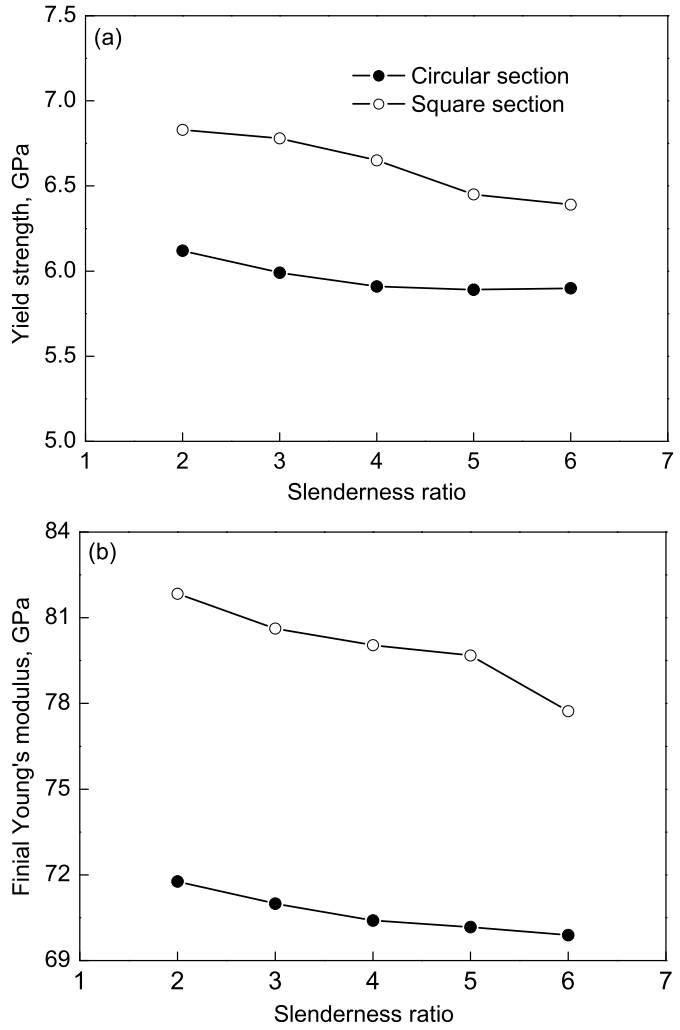

图 9 屈服强度和弹性模量随长细比变化曲线

Fig.9 Yield strength and Young's modulus as a function of slenderness ratio

$\begin{array}{ll}\text { (a) Yield strength } & \text { (b) Finial Young's modulus }\end{array}$ 
小规模的仿真结果略有不同 [6]. 这表明在小规模的仿真 条件下, 由于表面原子所占比例大, 表面效应对纳米杆的 内部位错形核及延展影响较大, 单独增加纳米杆的一维长 度, 纳米杆的整体密度并未发生改变, 因此, 长细比对纳 米杆力学特性的影响并不明显; 在大规模尺度下, 纳米杆 的表面原子比例大大减小, 表层原子位错形核后更易扩展, 表面效应的影响被削弱, 因此, 随着长细比的增大, 纳米杆 的屈服应力和弹性模量均略有下降, 表现为较好的塑性.

\section{5 结论}

本文利用分子动力学模拟了多种尺度下圆形、方形截 面形状的单晶 $\mathrm{Cu}$ 纳米杆的拉伸变形过程, 结论如下:

(1) 具有不同截面形状的单晶 $\mathrm{Cu}$ 纳米杆在弹性变形 阶段内部无位错形核, 变形为晶格尺寸的同比例放大; 屈 服阶段的初始塑性均表现为位错首先在纳米杆表面形核, 且位错形核发生在塑性流动之前; 首次屈服后, 纳米杆在 “位错形核 - 位错延伸与滑移 - 晶格原子交叉滑移” 的交 替循环的作用机制下，产生塑性变形; 断裂前期，“颈缩” 区的一部分晶格原子呈现非晶态.

(2) 截面形状对纳米杆拉伸力学特性有较大影响. 在 较小截面面积下, 方形截面形状纳米杆的弹性模量、屈服 应力都大于圆形截面形状纳米杆; 随着截面面积的增大, 不同截面形状纳米杆的屈服应力均减小, 屈服应变提前, 弹性模量增大; 当两种截面形状纳米杆的截面积增大到某 一定值后, 弹性模量趋近于其理论值.

(3) 大规模原子模拟表明, 长细比对纳米杆的力学特 性略有影响. 随着长细比的增大, 纳米杆的屈服应变提前, 屈服应力减小, 弹性模量出现 “软化” 现象.

\section{参考文献}

[1] Craihead H G. Science, 2000; 290: 1532

[2] Michalske T A, Houston J E. Acta Mater, 1998; 46: 391

[3] Greer J R, Oliver W C, Nix W D. Acta Mater, 2005; 53: 1821

[4] Komanduri R, Chandrasekaran R N, Raff L M. Science,
2001; 43: 2237

[5] Liang H Y, Ni X G, Wang X X. Acta Metall Sin, 2001; 37: 833

(梁海式, 倪向贵, 王秀喜. 金属学报, 2001; 37: 833)

[6] Liang H Y, Wang X X, Wu H G, Wang Y. Acta Mech Sin, 2002; 34: 208

(梁海七, 王秀喜, 吴恒安, 王 宇. 力学学报, 2002; 34: 208)

[7] Xu Z, Wang X X, Liang H Y. Chin J Mater Res, 2003; 17: 262

(徐 洲, 王秀喜, 梁海式. 材料研究学报, 2003; 17: 262)

[8] Doyama M, Kogure Y, Nozaki T, Kato Y. Phys Rev, 2003; 202B: 64

[9] Nozaki T, Masao D, Kogure Y. Mater Sci Eng, 2003; A350: 233

[10] Huang D, Tao W M, Guo Y M. Acta Mech Solid Sin, 2005; 26: 241

(黄 丹, 陶伟明, 郭乙木. 固体力学学报, 2005; 26: 241)

[11] Liang Y C, Chen J X, Bai Q S, Tang Y L, Chen M J. Acta Metall Sin, 2008; 44: 119

(梁迎春, 陈家轩, 白清顺, 唐玉兰, 陈明君. 金属学报, 2008; 44: 119)

[12] Tschopp M A, McDowell D L. J Mech Phys Solids, 2008; 56: 1806

[13] Zhang Y, Yang X H, Chen C Y. Acta Mech Solid Sin, 2009; 30: 231

(张 宇, 杨新华, 陈传尧. 固体力学学报, 2009; 30: 231)

[14] Daw M S, Baskes M I. Phys Rev, 1984; 29B: 8486

[15] Diao J K, Gall K, Dunn M L, Zimmerman J A. Acta Mater, 2006; 54: 643

[16] Swope W C, Anderson H C, Berens P H, Wilson K R. J Chem Phys, 1982; 76: 637

[17] Nose S A. J Chem Phys, 1984; 84: 511

[18] Hoover W G. Phys Rev, 1985; 31A: 1695

[19] Kelchner C L, Plimpton S J, Hamilton J C. Phys Rev, 1998; 58B: 11085

[20] Guo Yu, Zhuang Z, Li X Y. Chin J Theoretical and Appl Mech, 2006; 38: 398 (郭 宇, 庄 茁, 李晓雁. 力学学报, 2006; 38: 398)

[21] Hirth J P, Lothe J. Theory of Dislocations. New York: John Wiley and Sons, 1982: 757

[22] Mizubayashi H, Matsuno J, Tanimoto H. Scr Mater, 1999; 41: 443 